\title{
Clinical Profile of Child COVID-19 Patients of Bangladesh
}

\author{
Sayeeda Anwar ${ }^{1}$, Iffat Ara Shamsad ${ }^{1}$, AKM Amirul Morshed ${ }^{2}$, Fatema Farzana ${ }^{1}$ \\ ${ }^{1}$ Department of Paediatrics, Dhaka Medical College Hospital, Dhaka, Bangladesh \\ ${ }^{2}$ Department of Paediatric Hematology and Oncology, Dhaka Medical College Hospital, Dhaka, Bangladesh
}

\section{Email address:}

anwar_sayeeda@yahoo.com (S. Anwar)

\section{To cite this article:}

Sayeeda Anwar, Iffat Ara Shamsad, AKM Amirul Morshed, Fatema Farzana. Clinical Profile of Child COVID-19 Patients of Bangladesh. American Journal of Pediatrics. Vol. 7, No. 1, 2021, pp. 5-8. doi: 10.11648/j.ajp.20210701.12

Received: December 29, 2020; Accepted: February 3, 2021; Published: February 10, 2021

\begin{abstract}
Background: Millions of people worldwide have been affected by the COVID-19 pandemic. Due to the prevalence of malnutrition, refusal of immunization during a pandemic, nutritional anemia, air pollution, poverty, poor parental education, inadequate access to high-quality acute healthcare, the clinical characteristics and outcomes of children in Bangladesh may vary from other countries. Information on clinical presentations, outcomes, the relationship between disease incidence and the prevalence of associated disease in Bangladeshi children affected by COVID-19 are scarce. Objective: In this study, our main goal was to evaluate the clinical profile of pediatric COVID-19 patients in child corona unit of Dhaka Medical College Hospital, Bangladesh. Method: This single center observational study was conducted in Child Corona Unit of Dhaka Medical College Hospital (DMCH). A total of 1020 COVID-19 positive pediatric patients were included in this study. Results: In our study, $89.1 \%$ of patients had fever, $80.8 \%$ had a cough, $23.1 \%$ had diarrhea, and $70.8 \%$ had myalgia. According to CXR reports, $2.1 \%$ patients had ground-glass opacity, $38 \%$ had local patchy shadowing, $31.8 \%$ patients had bilateral patchy shadowing and $27.9 \%$ patients had interstitial abnormalities. Correlation of disease severity between without co-morbidity and with co-morbidity is statistically significant ( $\mathrm{p}=0.01$ ). Conclusion: This research revealed a variable range of presentations. This sheds light on the cases of COVID-19 in the pediatric population. Children with COVID-19 normally present with or are asymptomatic with different symptoms; infants may have a high risk of serious illness. However, most cases were reported in children 11-15 years of age and fever, cough, nasal congestion and dyspnoea were typical symptoms. Serious cases were those with co-morbidity and in order to save them additional attention during home care and prompt hospitalization therapy are needed.
\end{abstract}

Keywords: COVID-19 Pandemic, Clinical Profile, Pediatric Population

\section{Introduction}

The strain of coronavirus that causes coronavirus disease 2019 (COVID-19), the infectious disease responsible for the COVID-19 pandemic, is the extreme acute respiratory syndrome coronavirus 2 (SARS-CoV-2). It was previously referred to by its provisional name, 2019 novel coronavirus (2019-nCoV), and was also referred to as human coronavirus 2019 (HCoV-19 or hCoV-19) [1-4] The primary reports of COVID-19 infection noted the rarity of sickness in infants, including only 9 young people under the age of 14 . It has been shown that children with fluctuating degrees of severity are also affected by COVID-19. Epidemiological studies have consistently shown that children are at lower risk of producing severe side effects or opposing and adult simple sickness.
The 2019-nCoV has a near resemblance to bat coronaviruses, and the primary source of bats has been postulated. It spread rapidly through more than 85,000 individuals infecting China. Within a few months, Europe was engulfed, causing major losses of life and property in Italy, Spain, France, Germany, the United Kingdom and the United States. India, which is the second-most populous nation in the world, is now poised to gain a foothold. As of now, over 650,000 people across the globe have been infected and 28,000 people have succumbed to the disease. The epidemic was declared a public health emergency of international concern by the World Health Organisation on 30 January 2020, and a pandemic on 11 March 2020. [5, 6]

In March 2020, the virus was reported as having spread to Bangladesh. The country's epidemiology institute, IEDCR, announced the first three identified cases on 8 March 2020. 
The pandemic has since spread day by day across the entire nation and the number of people affected has risen. There were a total of 210,525 confirmed cases in the country as of 21 July 2020, with 114,870 recoveries and 2,709 deaths. [7] Even children are not out of this pandemic danger; the number of COVID-19 positive neonatal patients is also growing. Illness varies in severity from asymptomatic or moderate to severe; severe illness occurs in a large proportion of patients with clinically apparent infection. [8] We have conducted a study here and our main objective is to assess the clinical profile of pediatric COVID-19 patients in Bangladesh.

\section{Objective}

This study aims to assess the clinical profile of pediatric COVID-19 patients in Bangladesh.

\section{Methodology \& Materials}

This is a single center observational study done in Child Corona Unit of Dhaka Medical College Hospital. We identified all hospitalized children diagnosed with COVID - 19 from $10^{\text {th }}$ May to $17^{\text {th }}$ December, 2020. Realtime reverse transcriptase polymerase chain reaction (RTPCR) was performed on children admitted in Child corona unit. A total of 1020 COVID-19 patients with positive RTPCR were included in this study among 1403 suspected cases. A semi-structured questionnaire was used to obtain information including demographic information (age, sex, and geographic location), medical history, exposure history, clinical features (signs and symptoms at admission, dates of admission and diagnosis) were collected by attending physician. Clinical, laboratory, and radiological characteristics and treatment (any need of intensive care unit, oxygen therapy, antiviral therapy, antibiotics, corticosteroid therapy) and prognosis (any severe complications, including death), time from exposure to reverse transcription polymerase chain reaction (RT - PCR) negative, and discharge date etc data were obtained with data collection forms by the physician from medical records The data were reviewed by two physicians. All data was received through the complete consent of the guardian of the pediatric patients and hospital. Fever was defined as an axillary temperature of $37.5^{\circ} \mathrm{C}$ or higher. Collected data was collated and appropriate statistical analysis was done using computer-based SPSS (Statistical Package for the Social Sciences) version-20.

\section{Results}

Table 1 shows the demographic characteristics of the patients of our study. The male and female ratio was 1.53:1. The median age of the patients were presented in months. The distribution of age group shows that maximum patients $(30.6 \%)$ were in the group of $11-15$ years.
Table 1. Demographic profile of children in child corona unit of DMCH $(n=1020)$.

\begin{tabular}{lll}
\hline Characteristic & Number of patients & Percentage (\%) \\
\hline Male: Female & $1.53: 1$ & \\
Age, median (in months, IQR) & 79 & \\
Age group (year) & & \\
$<1$ & 182 & $18.2 \%$ \\
$1-5$ & 296 & $29.1 \%$ \\
$6-10$ & 226 & $22.1 \%$ \\
$11-15$ & 316 & $30.6 \%$ \\
\hline
\end{tabular}

Figure 1 shows the demographic profile (living place) of the COVID-19 positive children. Here we observed maximum patients were from Dhaka, Mymensingh and Narayanganj who were admitted in DMCH child corona unit.

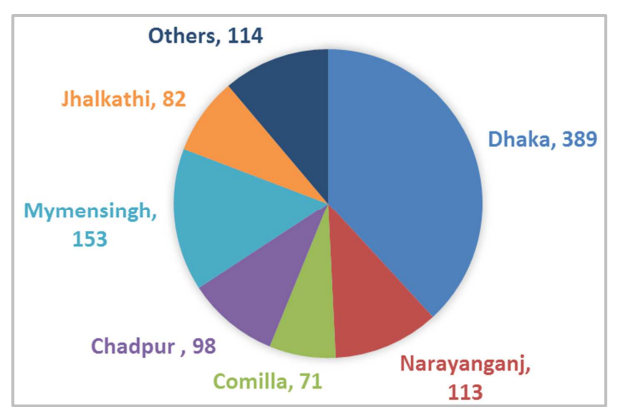

Figure 1. Demographic profile of children in child corona unit.

Table 2 shows the severity of the disease among all patients. Here maximum $(36.3 \%)$ patients were in severe condition.

Table 2. Disease severity and outcome of children in child corona unit of $\mathrm{DMCH}(n=1020)$.

\begin{tabular}{lll}
\hline Characteristic & Number of patients & Percentage (\%) \\
\hline Death & 42 & $4.1 \%$ \\
Severity of illness & & \\
Mild & 108 & $10.5 \%$ \\
Moderate & 311 & $30.5 \%$ \\
Severe & 370 & $36.3 \%$ \\
Critical & 231 & $22.7 \%$ \\
\hline
\end{tabular}

Table 3. Clinical Manifestations of Children in Child corona unit of DMCH $(n=1020)$.

\begin{tabular}{lll}
\hline Clinical Manifestations & Number & Percentage (\%) \\
\hline Fever & 909 & $89.1 \%$ \\
Nasal Congestion & 765 & $75 \%$ \\
Cough & 825 & $80.8 \%$ \\
Dyspnoea & 703 & $68.9 \%$ \\
Feeding problem & 348 & $34.1 \%$ \\
Abdominal pain / Vomiting & 152 & $14.9 \%$ \\
Diarrhea & 236 & $23.1 \%$ \\
Myalgia / Headache & 723 & $70.8 \%$ \\
Rash & 42 & $4.1 \%$ \\
Convulsion, Unconsciousness & 62 & $6 \%$ \\
Oxygen saturation <92\% during period & 663 & $65 \%$ \\
of hospitalization & & \\
Leukopenia/ Lymphopenia/High CRP & 799 & $78.3 \%$ \\
CXR & & \\
Ground-glass opacity & 22 & $2.1 \%$ \\
Local patchy shadowing & 388 & $38 \%$ \\
Bilateral patchy shadowing & 325 & $31.8 \%$ \\
Interstitial abnormalities & 285 & $27.9 \%$ \\
\hline
\end{tabular}


The clinical profile of all patients are presented in table 3 which shows fever, nasal congestion, cough, myalgia, leukopenia/lymphopenia were very common symptoms and maximum patients had these.

The table 4 of disease severity between without comorbidity and with co-morbidity we found the $\mathrm{P}$-value $=0.01$, which is less than 0.05 . So the correlation of disease severity between without co-morbidity and with co-morbidity is statistically significant.

Table 4. Distribution of disease severity, mortality among children in child corona unit $(n=1020)$.

\begin{tabular}{llll}
\hline Disease severity & Without co-morbidity & With co-morbidity & P value \\
\hline Mild & 45 & 67 & \\
Moderate & 78 & 122 & \\
Severe & 82 & 143 & $\mathrm{P}=0.01$ \\
Critical & 51 & 92 & \\
Death & 3 & 18 & \\
\hline
\end{tabular}

In the figure 2, the data of child corona unit's patients profile are shown. Here it shows maximum patients were discharged after proper treatment.

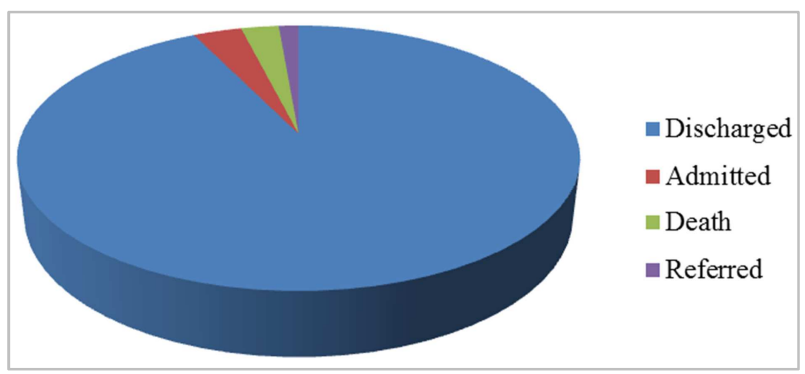

Figure 2. Patient Profile of children in Corona Care unit $(n=1020)$.

\section{Discussion}

In our study, we distributed patients between the age group of $<1-15$ years. And the maximum (30.6\%) number of patients were 11-15 years old. In this study, most patients who had COVID-19 were male. The immune cells are more susceptible to damage by the virus with growing age, and therefore their immunity may be diminished. [11] These findings were similar to those seen in adults and were either asymptomatic or moderate in most pediatric patients with these outcomes. [13] We also found that $89.1 \%$ of patients had a fever, $80.8 \%$ had a cough, $23.1 \%$ had diarrhea, and $70.8 \%$ had myalgia. According to a report, fever and cough were present in the children (50\%) studied, much like the first signs of adult infection.[13] Another study also revealed that the most common symptom presented in patients was cough $(85.71 \%)$ accompanied by fever $(78.57 \%)$ [9, 10], which was the most common symptom observed in fever (91.7\%) in comparison to literature (91.7\%). [12]

In our study CXR of the patients showed, $2.1 \%$ patients were undergone Ground-glass opacity, $38 \%$ were undergone local patchy shadowing, $31.8 \%$ patients were undergone Bilateral patchy shadowing and $27.9 \%$ patients had interstitial abnormalities. Early chest CT results of children with COVID-19 have certain parallels with those of adults but differ in their ways. Compared to females, lymphopenia was much more often seen in male patients. Lymphopenia suggested that the presence of lymphopenia can be used as a marker of prognosis for all patients needing oxygen support. [9] In our study, the disease severity between without comorbidity and with co-morbidity we found the P-value $=0.01$, which is less than 0.05 . It showed that the correlation of disease severity between without co-morbidity and with comorbidity wass statistically significant.

Subsequently, we are reminded that pediatric infection prevention and management is also a family endeavor. Both basic but significant preventive measures are respiratory isolation, hand hygiene, and surface disinfection. It is very impractical for infants or toddlers to donate masks, aside from the current lack of medical masks appropriate for children, and many older children are unable to selfdiscipline in viral war. Parents must then do the same for their children when tending to their obligations, focusing on respiratory protection and hand hygiene.

\section{Conclusion}

The COVID-19 cases in the pediatric population were represented in this report. A high risk of serious illness can be present in children with COVID-19 who normally have different symptoms or are asymptomatic. In order to stop and track this pandemic, we must pay attention to the many children who are asymptomatic carriers. However, most cases were reported in children 11-15 years of age and fever, cough, nasal congestion and dyspnoea were typical symptoms. Serious cases were those with co-morbidity and in order to save them additional attention during home care and prompt hospitalization therapy are needed. For treatment guidelines and testing protocols, further research is needed.

\section{References}

[1] Gorbalenya AE, Baker SC, Baric RS, de Groot RJ, Drosten C, Gulyaeva AA, et al. (March 2020). "The species, severe acute respiratory syndrome-related coronavirus: classifying 2019-nCoV and naming it SARS-CoV-2". Nature Microbiology. 5 (4).

[2] "Coronavirus disease named Covid-19". BBC News Online. 11 February 2020 . Archived from the original on 15 February 2020. Retrieved 15 February 2020.

[3] Surveillance case definitions for human infection with novel coronavirus (nCoV): interim guidance v1, January 2020 (Report). World Health Organization. January 2020. hdl: 10665/330376. WHO/2019-nCoV/Surveillance/v2020.1.

[4] Healthcare Professionals: Frequently Asked Questions and Answers". United States Centers for Disease Control and Prevention (CDC). 11 February 2020. Archived from the original on 14 February 2020. Retrieved 15 February 2020.

[5] Guan W, Ni Z., Hu Y, Liang W, Ou C, He J. Clinical Characteristics of Coronavirus Disease 2019 in China. N Engl J Med 2020; 382: 18 nejm.org. 
[6] Lei H, Li Y, Xiao S, et al. Routes of transmission of influenza A H1N1, SARS CoV, and norovirus in air cabin: comparative analyses. Indoor Air 2018; 28: 394-403.

[7] Research (IEDCR), Institute of Epidemiology, Disease Control and. Research, corona.gov.bd (in Bengali). Retrieved 3 May 2020.

[8] Na Zhou et al: A novel coronavirus from patients with pneumonia in China, 2019. N Engl J Med. 2020 Feb 20.

[9] Bhandari, S., Bhargava, A., Sharma, S., Keshwani, P., Sharma, R., \& Banerjee, S. (2020). Clinical profile of COVID-19 infected patients admitted in a tertiary care hospital in north India. J Assoc Physicians India, 68, 13-17.

[10] X. F. Lu, W. Gong, L. Wang, et al., Clinical features and high- resolution $\mathrm{CT}$ imaging findings of preliminary diagnosis novel coronavirus pneumonia, Zhonghuafangshexuezazhi 54 (00) (2020) E007, https://doi.org/10.3760/cma.j. ISSN. 10051201.2020.0007.

[11] Liu WJ, Zhao M, Liu K, Xu K, Wong G, Tan W, Gao GF. Tcell immunity of SARS-CoV: implications for vaccine development against MERS-CoV. Antiviral Res 2017; 137: 82-92.

[12] Huang C. Wang Y, Li X et al. Clinical features of patients infected with 2019 novel coronavirus in 312 Wuhan, China, Lancet 2020.

[13] Dong Y, Mo X, Hu Y, et al. Epidemiology of COVID-19 among children in China. Pediatrics. 2020; 145: e2 0200702. 